\title{
Reflets
}

Revue d'intervention sociale et communautaire

\section{Les soins de santé des familles haïtiennes de Montréal face aux maux de ventre de leurs enfants}

\section{Liliana Gomez Cardona}

Volume 17, numéro 2, automne 2011

Représentations, pratiques et expérience dans le champ de santé : tendre des ponts entre des disciplines

URI : https://id.erudit.org/iderudit/1012131ar

DOI : https://doi.org/10.7202/1012131ar

Aller au sommaire du numéro

Éditeur(s)

Reflets, Revue d'intervention sociale et communautaire

ISSN

1203-4576 (imprimé)

1712-8498 (numérique)

Découvrir la revue

Citer cet article

Gomez Cardona, L. (2011). Les soins de santé des familles haïtiennes de Montréal face aux maux de ventre de leurs enfants. Reflets, 17(2), 101-126. https://doi.org/10.7202/1012131ar

\section{Résumé de l'article}

Cet article fait la suite à une recherche de terrain menée auprès de migrants haïtiens de Montréal et dont l'objectif était de comprendre le faible taux des familles immigrantes qui consultent le département de gastroentérologie du CHU Ste-Justine. Nous nous sommes intéressée aux immigrants haïtiens parce qu'ils sont porteurs d'une culture médicale importante et constituent une des minorités les plus nombreuses à Montréal. La démarche méthodologique adoptée a été qualitative, selon une approche anthropologique de type herméneutique. L'analyse s'est basée sur le modèle "signes, sens, actions " (Corin et Bibeau, 1995). Par le biais de treize entretiens semi-dirigés menés auprès de cinq familles haïtiennes, nous documentons les explications et les moyens de soulagement des maux de ventre chez leurs enfants. De même, nous identifions les facteurs qui amènent ces familles à effectuer ou non des démarches de recherche d'aide auprès des services cliniques, et dégageons quelques pistes de réflexion utiles aux cliniciens et intervenants qui travaillent avec cette population.
Tous droits réservés $@$ C Reflets, Revue d’intervention sociale et communautaire, 2012
Ce document est protégé par la loi sur le droit d'auteur. L'utilisation des services d'Érudit (y compris la reproduction) est assujettie à sa politique d'utilisation que vous pouvez consulter en ligne. 


\title{
Les soins de santé des familles haïtiennes de Montréal face aux maux de ventre de leurs enfants
}

\author{
Liliana Gomez Cardona \\ M. Sc. Université de Montréal
}

\section{Résumé}

Cet article fait la suite à une recherche de terrain menée auprès de migrants haïtiens de Montréal et dont l'objectif était de comprendre le faible taux des familles immigrantes qui consultent le département de gastroentérologie du CHU Ste-Justine. Nous nous sommes intéressée aux immigrants haïtiens parce qu'ils sont porteurs d'une culture médicale importante et constituent une des minorités les plus nombreuses à Montréal. La démarche méthodologique adoptée a été qualitative, selon une approche anthropologique de type herméneutique. L'analyse s'est basée sur le modèle "signes, sens, actions " (Corin et Bibeau, 1995). Par le biais de treize entretiens semi-dirigés menés auprès de cinq familles haïtiennes, nous documentons les explications et les moyens de soulagement des maux de ventre chez leurs enfants. De même, nous identifions les facteurs qui amènent ces familles à effectuer ou non des démarches de recherche d'aide auprès des services cliniques, et dégageons quelques pistes de réflexion utiles aux cliniciens et intervenants qui travaillent avec cette population.

Mots clés : Haïtiens, immigrants, alliance thérapeutique, soins de santé, anthropologie de la santé, maux de ventre. 
This article is the result of a field research conducted among Haitian immigrants in Montreal, whose objective was to understand the low rate of immigrant families who visit the Gastroenterology Department of CHU Ste-Justine. We were interested in Haitian immigrants because they have an important medical culture and are one of the largest minorities in Montreal. We followed a qualitative methodology based on anthropological and hermeneutical approaches through thirteen semi-structured interviews conducted with five Haitian families. The analysis was based on the model "signs, meanings and actions " (Corin et Bibeau 1995). In this article we document the explanations and means of relieving the stomachaches of their children. Similarly, we identify the factors that led these families to do - or not - efforts to seek help from clinical services, and disclaim some useful ideas to clinicians who work with these populations. In general, these families assign tentative explanations for aches. However, they are in constant reformulation. Regarding the resources to deal with stomachaches, these families use different and simultaneous methods. The family space, church activities and links to community organizations are privileged therapeutic spaces. In general these families have ambivalent opinions about medical services and consider them as their last resource.

Key words : Haitians, immigrants, therapeutic alliance, health care, medical anthropology, stomachache/bellyache.

\section{Introduction}

Très peu d'auteurs se sont penchés sur la santé des immigrants d'origine haïtienne à Montréal dans une perspective qui laisse place à leur discours. Les travaux sur les principaux problèmes de santé affectant cette population remontent aux années 1980 et 1990. Quelques-uns de ces travaux ont signalé les principaux motifs de consultation médicale, tels que les désordres psychosomatiques et 
"...les migrants haïtiens tendent à utiliser très peu les services de santé biomédicaux..."

"...ils ont recours, de ressources qu'ils utilisent ou d'idée qu'ils se font de la santé et de la maladie. " fonctionnels, les problèmes de nutrition, la tuberculose, l'anémie falciforme, les problèmes d'adaptation scolaire et la violence intrafamiliale (Pierre-Jacques, 1982; 1980). D'autres études ont indiqué que les migrants haïtiens tendent à utiliser très peu les services de santé biomédicaux de la société québécoise (Bibeau, 1982; Massé, 1995). Une étude plus récente, réalisée par l'Institut de la statistique du Québec et par le ministère de la Santé et des Services sociaux portant sur l'état de santé et les caractéristiques sociodémographiques des immigrants haïtiens arrivés à Montréal entre 1988 et 1997 a également démontré que cette population accède moins aux services de santé et aux consultations médicales que le reste de la population québécoise. Pourtant, des études indiquent que ces immigrants utilisent autant les services de soins de santé institutionnalisés que d'autres options thérapeutiques alternatives pour se soigner (Bibeau, 1987; Massé, 1995; Brodwin, 1996). Les motifs de cette situation demeurent inconnus (Institut de la statistique du Québec et ministère de la Santé et des Services sociaux, 2006). Il en est de même des habitudes des migrants haïtiens en termes de soins de santé auxquels ils ont recours, de ressources qu'ils utilisent ou d'idée qu'ils se font de la santé et de la maladie. Tout comme font défaut les évaluations systématiques sur l'état de santé de cette population. On note également un manque d'études illustrant le point de vue des familles, des parents et des enfants sur la santé et les maladies des plus petits.

La majorité des études ayant abordé la question de la santé chez les immigrants haïtiens à Montréal sont ancrées dans une perspective épidémiologique ou psychologique (Bijoux, 1997). Très peu de recherches anthropologiques ont été réalisées sur le sujet. Parmi les apports les plus récents qui tiennent compte autant des données épidémiologiques que des données qualitatives, on trouve l'analyse de Massé (1995) sur les principaux facteurs qui influencent la santé des immigrants haïtiens à Montréal. Qu'ils soient prémigratoires ou postmigratoires, ces facteurs peuvent selon Massé agir en tant que source de protection ou encore de vulnérabilité, dépendant des conditions concrètes de la vie de chaque individu. Quelques études qui ont aussi été réalisées en sciences humaines appliquées et en travail social.Elles s'intéressent 
«Nous nous

intéressons ici aux

représentations

qu'entretiennent les

familles haïtiennes à

Montréal à l'égard des soins de santé et des maux de ventre chez les enfants. " surtout aux aspects liés à la structure familiale des immigrants haïtiens à Montréal (Argant-Le Clair, 1991; Claire, 2005; Compere et Craan, 1997).

Si on considère l'importance de l'immigration haïtienne au Québec, selon le recensement de 2006, les Noirs formaient avec 188100 personnes la plus grande minorité visible de la province - 52,5\% de ces dernières ayant Haïti comme pays de naissance. Ces personnes forment également la première minorité visible en importance dans la région métropolitaine de Montréal - 169100 personnes. Environ 55,9 \% des Noirs de Montréal sont nés à l'étranger, et plus de la moitié de ceux-ci, soit 55,4 \%, sont natifs d'Haïti (Statistique Canada, 2009). C'est pourquoi une meilleure connaissance des représentations et des comportements des immigrants haïtiens par rapport à la santé nous semble nécessaire.

Dans cette perspective, cet article présente les résultats d'une recherche de terrain menée dans le cadre d'une maitrise en anthropologie et qui avait pour but de comprendre les perceptions, les explications et les moyens de soulagement des maux de ventre chez les enfants des familles haïtiennes à Montréal. Cette recherche s'inscrit dans le cadre d'une étude plus large, dans laquelle nous nous proposons de répondre, entre autres questions, à celle concernant le faible taux de familles immigrantes qui consultent la clinique de gastro-entérologie du CHU Ste-Justine au sujet des maux de ventre de leurs enfants. Nous nous intéressons ici aux représentations qu'entretiennent les familles haïtiennes à Montréal à l'égard des soins de santé et des maux de ventre chez les enfants. Nous souhaitons circonscrire les explications et les réponses données à ces douleurs, à la lumière des rapports sociaux reconstruits à travers la migration, des structures et des dynamiques de familles haïtiennes de Montréal touchées par notre étude.

D'un autre côté, des données épidémiologiques suggèrent que les taux de prévalence des désordres fonctionnels de l'intestin augmentent un peu partout dans le monde (Okeke, et collab., 2005; Olubuyide, et collab., 1995 ). Bien que des études signalent des facteurs psychosociaux de risque comme le stress 
"...qu'une approche anthropologique centrée sur l'expérience des enfants et de leurs familles se veut pertinente et enrichissante quant à la compréhension des problèmes gastriques. "

"Les immigrants d'origine

haitienne ... sont porteurs de traditions différentes de celles $d u$ groupe majoritaire..."
(Levy, et collab., 2006), les différences culturelles sur les plans de la perception, de l'interprétation des symptômes et du seuil de tolérance à la douleur (Masud, et collab., 2001; Talley et Spiller, 1991) sont très peu connues. Aussi, l'influence de la famille dans le modelage et le renforcement des comportements de l'enfant face à la maladie (Kanasawa, et collab., 2004; Lindley, et collab., 2005) tout comme les autres dimensions socioculturelles associées à ce type de maladie restent encore peu explorées.

L'épidémiologie met surtout l'accent sur l'influence du comportement individuel sur la santé et la maladie, négligeant quelque peu les dimensions sociales et culturelles. C'est dans ce sens qu'une approche anthropologique centrée sur l'expérience des enfants et de leurs familles se veut pertinente et enrichissante quant à la compréhension des problèmes gastriques. Dans ce contexte, lorsqu'on s'intéresse aux maux de ventre des enfants, on est amené à mettre entre parenthèses la nosographie et l'étiologie médicale courante, pour examiner les manières dont les enfants et leurs parents décrivent les symptômes, et leur donnent une forme en mobilisant les mots qui sont signifiants pour eux. À travers cette approche, c'est toute une problématique de l'expérience qui se découvre, dans la foulée de ce que l'on pourrait appeler une alternative anthropologique à une vision plus standardisée du corps. Une telle alternative consiste à se dégager provisoirement des modèles posés en extériorité de l'expérience, pour examiner ce que Kleinman (1988) appelle «l'expérience personnelle de la maladie ", telle qu'elle se propose à travers les récits des personnes rencontrées dans leur milieu de vie : une expérience dans laquelle se tissent d'une manière complexe des éléments du monde social, de la subjectivité et des processus psychophysiologiques.

Les immigrants d'origine haïtienne représentent un groupe d'étude intéressant, non seulement parce qu'ils constituent une des minorités visibles les plus nombreuses à Montréal, mais parce qu'ils sont porteurs de traditions différentes de celles du groupe majoritaire local. Cela est particulièrement vrai en ce qui concerne les modalités d'appropriation du corps, les manières de penser et de traiter les maladies, tout comme d'autres aspects de la socialisation (Brodwin, 1996; Laguerre, 1987). De plus, bien que 
les Haïtiens en contexte migratoire suscitent un intérêt particulier chez les professionnels de la santé publique, de la médecine et de l'anthropologie depuis quelques décennies, notamment en ce qui concerne leurs catégories étiologiques (Craan, 1991; Massé, 1995), il n'existe pas d'étude sur les problèmes de ventre, ni d'étude qui prennent en compte les points de vue des familles et de leurs enfants en dehors du contexte clinique.

\section{Indications méthodologiques}

"...approfondir le

sens des données

et d'en respecter

la complexité, en

soulignant la nature

sociale et culturelle des

processus de maladie."

"Au-delà de la diversité de structures des maisonnées, les familles rencontrées avaient en commun une caractéristique importante : en tant que chefs de la maison, les mères jouent un rôle important au sein de leurs familles nucléaires. »
La démarche méthodologique adoptée est qualitative, d'inspiration anthropologique avec une perspective sémiologique et interprétative. Elle permet d'atteindre et de documenter du point de vue des familles elles-mêmes les représentations et les réactions face aux maux du ventre. Contrairement aux recherches quantitatives, qui mettent l'accent sur la quantité de données accumulées et sur la mesure et l'analyse des relations causales entre variables (Denzin et Lincoln, 2000, p. 8), cette perspective méthodologique permet d'approfondir le sens des données et d'en respecter la complexité, en soulignant la nature sociale et culturelle des processus de maladie. Par ailleurs, nous n'avons pas cherché à atteindre une saturation théorique de l'échantillon, mais plutôt à créer une analyse intensive autour du matériel empirique.

L'échantillon a été composé de cinq unités familiales à composition variable répondant à quelques critères généraux : il s'agit de parents nés en Haiti, ayant émigré au Canada et vivant actuellement à Montréal, ayant un ou plusieurs enfants, filles ou garçons, âgés de 8 à 16 ans, lesquels ont fréquemment des maux de ventre. Ces enfants ne devaient pas nécessairement être nés en Haiti. Nous avons rencontré deux familles monoparentales, deux familles biparentales, dont une recomposée, et une famille dont le mari demeure toujours en Haïti. Au-delà de la diversité de structures des maisonnées, les familles rencontrées avaient en commun une caractéristique importante : en tant que chefs de la maison, les mères jouent un rôle important au sein de leurs familles nucléaires. De plus, la famille nucléaire agit comme un 
lieu privilégié d'investissement affectif, de soutien, d'appartenance et de socialisation de ses membres. En ce qui concerne les conditions économiques et de vie, ces mères ont tendance à se percevoir dans des conditions plutôt précaires. Au chapitre des motifs d'immigration, toutes les mères disent avoir immigré en raison des conditions économiques difficiles et de l'insécurité en Haïti.Trois familles ont leur citoyenneté canadienne, une famille est résidente et une autre famille a demandé l'asile.

$\mathrm{Au}$ total, treize entretiens semi-dirigés ont été réalisés, dont cinq entretiens familiaux - avec l'enfant et au moins un de ses parents - et huit entretiens individuels avec les enfants. La collecte des données a été réalisée de novembre 2008 à juin 2009. L'entretien semi-dirigé a été la technique privilégiée, puisqu'elle est appropriée pour appréhender de manière approfondie l'expérience des autres et pour connaitre du point de vue des acteurs sociaux eux-mêmes les sens et les pratiques liés à ces expériences (Mayer et Ouellet, 2000; Poupart, et collab., 1997). Les thèmes situés au centre de l'étude ont été : l'apparition et l'évolution du mal de ventre chez les enfants; la perception et l'expression des symptômes qui lui sont associés; les explications et les significations attribuées à ces symptômes; les réactions et les façons de gérer ces désordres; la place et l'impact que les maux ont sur la vie familiale et sur celle des enfants; les rapports des familles et des enfants avec les services médicaux; l'histoire de la famille; son parcours migratoire; et l'état de santé des autres membres de la famille.

Afin d'atteindre nos objectifs, nous avons pris appui, sur le modèle «signes, sens, actions » élaboré par Corin et Bibeau (1995), lequel est spécialement approprié lorsque l'on s'interroge sur les facteurs qui amènent certaines familles à recourir ou non à des services spécialisés. Nous avons exploré les signes, soit la perception et l'expression des symptômes et autres signes reliés aux troubles fonctionnels, du point de vue de l'enfant et de ses parents. Nous avons également identifié les sens attribués aux symptômes ainsi que les modèles d'explication soulevés par les différents acteurs - enfants et parents - et les contextes socioculturels plus larges autour desquels ils s'articulent. Enfin, nous avons examiné les 
actions associées aux désordres intestinaux chez les enfants, leurs parents et l'entourage, en particulier le traitement des symptômes et la recherche - ou non-recherche - d'aide. L'ensemble des variables sémiologiques — «signes " - recueillies a été mis en relation avec les variables d'interprétation - "sens »-, qui concernent la manière dont les enfants, leurs parents et leur entourage interprètent les symptômes. Enfin, ces variables ont été mises en relation avec les variables pragmatiques — " actions " -, qui regroupent les différents recours utilisés en présence des douleurs et malaises de l'enfant. L'analyse dynamique de ces trois catégories de variables a été menée à un niveau individuel afin de dégager du contenu des entretiens les thèmes émergents et récurrents des récits des enfants et des parents.

Cette démarche méthodologique nous a permis d'analyser la façon selon laquelle les enfants et les personnes de leur entourage familial élaborent l'expérience de cette "maladie " - les maux de ventre - et lui donnent un sens, en utilisant certains repères conceptuels et expressifs. De même, nous avons pu analyser la manière selon laquelle ces sens et signes se traduisent en manières de ressentir, d'exprimer et d'agir face à ces douleurs. Seule une démarche de recherche orientée vers une interprétation des conceptions que les familles se font des problèmes gastriques, et vers une lecture contextualisée - socialement et historiquement — de la culture des familles haïtiennes immigrantes nous a semblé apte à répondre à nos préoccupations.

\section{Résultats}

\section{Les symptômes des maux de ventre}

Les enfants et les parents présentent les maux de ventre comme étant irréguliers dans leur fréquence et leur intensité. Chez la majorité des enfants, ces maux coexistent avec d'autres malaises physiques comme la constipation et les maux de tête. De plus, différents sentiments ou émotions se mêlent intimement aux symptômes physiques. De manière générale, les symptômes apparaissent difficiles à décrire et à mesurer de façon sûre et 
méthodique. Une fille évoque sa difficulté à transmettre sa douleur aux médecins : "Personne ne veut vraiment savoir ce que j'ai... Les médecins me disent : «Tu as mal combien? «.Tu sais, le combien, pour eux c'est significatif, mais pour moi..." (Cathérine ${ }^{1}, 12$ ans). Ce caractère souvent imprécis entraine chez tous les enfants l'utilisation de qualificatifs et de métaphores qui témoignent de l'importance des maux.

\section{Les tentatives d'explication des maux de ventre}

"...on peut dégager quatre pôles autour desquels s'organisent leurs réponses:

l'alimentation; les virus; le froid; et les tensions sociales. ”
Les sens attribués aux maux de ventre sont variés. Les familles haïtiennes ont parlé d'un ensemble de facteurs pouvant les provoquer. Cependant, un tel ensemble ne constitue pas un schème statique à partir duquel elles donnent toujours une explication concluante sur les maux de ventre. Ce sont plutôt des explications plausibles, des tentatives d'explication qui agissent comme des pistes devant toujours être soumises à évaluation. Cette incertitude se traduit par le besoin de trouver une explication satisfaisante et amène les personnes à réfléchir et à vérifier, à chaque épisode de mal de ventre, quelles seraient les causes en jeu.

D'une manière générale, on peut dégager quatre pôles autour desquels s'organisent leurs réponses : l'alimentation; les virus; le froid; et les tensions sociales. On trouve aussi au sein de chaque famille différentes perspectives quant à la source des maux de ventre. Une telle diversité de points de vue témoigne de différentes conceptions de la nature de ces maux : le sens qui leur est associé varie considérablement d'un membre de la famille à un autre et ne se présente pas comme une entité fixe, mais plutôt comme le résultat d'un ensemble de facteurs, de contextes susceptibles d'affecter l'interprétation qui sera faite des symptômes observés.

\section{L'alimentation}

L'alimentation est souvent évoquée pour expliquer les symptômes et est considérée comme une source importante du problème. Ce sujet revêt différents aspects et est ordonné d'après différentes significations. Ainsi, selon certains individus, le fait de ne pas manger suffisamment, trop manger, de manger certains aliments ou encore de manger de la nourriture à une certaine température 
«...des relations tendues s'installent entre les mères et les enfants avec l'alimentation comme toile de fond, sa régulation, et les résistances et habitudes alimentaires déréglées des enfants. » occasionnerait des maux de ventre. Les mères ont mentionné le fait de manger certains aliments - dont le chou, le chou-fleur, le brocoli, le maïs moulu et les sucreries - comme une des principales causes des maux de ventre chez leurs enfants :

«Le plus souvent, c'est des friandises... Les enfants, ils aiment beaucoup les friandises. Quand on se lève, on n'a pas l'habitude de prendre des choses sucrées... parce que ça aide les maux de ventre, ça encourage, ça précipite les maux de ventre. " (mère de la famille Roney)

Dans la plupart des cas, elles ont associé ces maux de ventre à des gaz, lesquels peuvent être identifiés par le fait qu'on sent et peut palper des mouvements et du bruit dans le ventre :

«On a souvent des gaz qui bouillent au ventre. On sent que ça marche. On sent que ça fait du bruit, et puis ça provoque aussi des maux de ventre. Ça donne aussi de la diarrhée... Si on sent des palpitations parfois, et ça marche, c'est une crampe qui passe. Si la douleur est très forte, et puis ça ne passe pas, alors on pourrait dire que c'est du gaz.» (mère de la famille Pétrus)

De plus, des relations tendues s'installent entre les mères et les enfants avec l'alimentation comme toile de fond, sa régulation, et les résistances et habitudes alimentaires déréglées des enfants. Il en serait ainsi pour Louis, douze ans, qui n'aime pas manger la nourriture que sa mère lui prépare pour l'école. De même, les sœurs Catherine et Victoire mangent des sucreries le matin, malgré l'interdiction de leur mère et le fait qu'elles pensent que cela leur donnera des maux de ventre.

Une tendance marquée chez la plupart des enfants est de ne pas avoir de bonnes habitudes alimentaires. Ce sont surtout les mères qui se montrent les plus inquiètes au sujet de l'alimentation des jeunes et qui veulent gérer leurs habitudes alimentaires. Enfin, les enfants de la famille Pétrus ont rapporté avoir des rapports conflictuels avec la nourriture haïtienne et la percevoir comme 
"S'ils ont le choix, ils préferent manger des sandwichs. »

"Les maladies transmises par voie de contagion ou de contamination occupent une place importante..." une des causes possibles de leurs maux de ventre. À ce propos, Christine, fille aînée de douze ans, a dit ne pas aimer la nourriture haïtienne et ne pas bien la digérer, de sorte qu'elle sent qu'elle peut lui causer des maux de ventre. Son frère Augustin partage son opinion. Les deux ajoutent ne pas aimer la nourriture de l'école, notamment les plats qu'ils nomment " végétariens » ou "pour des Arabes ». S'ils ont le choix, ils préferent manger des sandwichs.

\section{Les virus}

Les maladies transmises par voie de contagion ou de contamination occupent une place importante dans les explications fournies par quelques familles participantes, les principales étant la gastroentérite et la malaria, lesquelles sont associées à des environnements quotidiens tels l'école et le transport en commun. Les familles participantes ont également mentionné des microbes qui se propagent par différents moyens, tels l'air, l'eau du robinet ou la viande de porc:

«Le plus souvent, on attribue ça à la race noire... On a dit [que ça provient] de la viande de porc. Ça donne beaucoup de vers. Mais moi, je ne la consomme presque pas. Chez les enfants, on a souvent dit que c'est héréditaire. Par exemple, si la maman a des vers, alors immédiatement, l'enfant en a.» (mère de la famille Cadette)

À cette notion de contamination biologique s'ajoutent parfois des sentiments de vulnérabilité et de discrimination chez ces familles qui peuvent se percevoir comme fragiles et "polluantes " pour leur environnement naturel et social :

"Le problème, c'est tellement attrapant que si un est attrapé, moi je sais que les trois sont attrapés. Parce qu'on ne peut pas les séparer, ils jouent, ils sont ensemble, ils sont à la maison. Les jumeaux, quand un a quelque chose, ça ne prend pas beaucoup de temps pour que l'autre attrape. C'est attrapant. » (mère de la famille Pétrus) 


\section{Le froid}

\section{"La température} froide est considérée par plusieurs familles comme une source de douleurs d'estomac. "
La température froide est considérée par plusieurs familles comme une source de douleurs d'estomac. Un tel facteur peut agir de différentes façons, que ce soit en se mettant en contact avec des objets froids ou des espaces froids, en consommant de la nourriture froide ou des boissons froides :

«Moi, j’ai pensé que c'était à cause que mes pieds. Je les mettais nus au-delà du sol, qui faisait froid... J'ai pensé que c'était à cause de ça, quand je marche pieds nus à la maison... quand la neige est glacée, et puis je marche.» (Catherine, douze ans)

La mère de cette famille partage le point de vue de sa fille et ajoute une remarque dont elle a entendu parler dans son milieu social d'origine :

"Quand une jeune fille allait se former, et si elles ont l'habitude de mettre les pieds par terre comme ça, elles pourraient attraper du froid qui pourrait entrainer encore des maux de ventre. Le mal au ventre prémenstruel est attribué à des négligences qu'on a l'habitude de faire au moment de l'adolescence... et puis ça peut donner de la suite à l'âge adulte et à la vieillesse aussi. »

Le refroidissement corporel est parfois désigné par le mot créole «fred» :

«On dit souvent en Haïti que [les personnes ont] de fred. "Fred, ça veut dire qu' [elles ont] du froid, qu'[elles ont] des symptômes de maux de ventre... qu'elles ont attrapé par des négligences, qu'elles sont restées avec des vêtements mouillés très longtemps, ou qu'elles ont laissé les pieds par terre de bon matin. On dit ça souvent. " (mère de la famille Pétrus) 
Les mères sont les répondantes ayant principalement utilisé ce terme. Les enfants n'ont presque jamais utilisé le mot "fred ", même si quelques-uns ont fait référence au froid comme une des causes de leurs maux de ventre.

\section{Les tensions sociales}

Enfin, quelques enfants soulignent que les tensions à l'école et les conflits avec d'autres personnes forment des sources possibles de leurs maux de ventre :

"C'était un mauvais moment, parce que je ne pouvais pas travailler, à cause qu'en première année et en deuxième année, je ne savais pas comment écrire, je ne savais pas comment lire. Puis, à plusieurs fois de redoubler ma deuxième année, ils m'ont fait former en troisième année... Cette année, j'ai eu une fois la gastro à l'école, et une fois à la maison. Quand j'étais entrée en classe, je commençais à travailler, mais j'avais des crampes.» (Caméla, onze ans)

"...leurs mères ont tendance à lier ces maux de ventre aux conditions sociales générales qui entourent leurs familles, comme le processus migratoire et la situation économique. "
En revanche, leurs mères ont tendance à lier ces maux de ventre aux conditions sociales générales qui entourent leurs familles, comme le processus migratoire et la situation économique. Les interviews témoignent de deux tendances principales en ce qui a trait à la perception du processus migratoire comme possible cause des maux de ventre chez les enfants et du mauvais état de santé de la famille en général. D'un côté, quelques familles ont remarqué une diminution de l'intensité et de la fréquence des maux de ventre chez les filles depuis leur arrivée au Canada. D'un autre côté, certaines personnes jugent que les conditions de vie après leur migration ont eu des effets néfastes sur leur état de santé. À ce propos, la mère de la famille Pétrus a parlé de la solitude et des difficultés économiques qu'elle a connues à son arrivée à Montréal; elles auraient causé les problèmes du ventre de ses trois enfants - entre autres problèmes de santé vécus par la famille. 


\section{Les ressources utilisées pour soulager les maux de ventre}

"L'espace familial est le principal espace thérapeutique où la plupart des enfants cherchent et trouvent des soins et $d u$ soulagement à leurs maux de ventre."
En général, ces familles développent des stratégies faisant appel à plusieurs ressources de santé. Ces dernières sont utilisées d'une manière parallèle ou complémentaire et se caractérisent par des soins entrepris dans l'espace familial, par des pratiques suivies au sein des églises fréquentées ou par des conseils prodigués par quelques proches. Ainsi, on voit que le champ d'action de la recherche d'aide et de solution aux problèmes de santé couvre non seulement les itinéraires de recherche d'aide formelle, mais également les stratégies internes à la famille et à la communauté. Les trajectoires thérapeutiques de ces familles sont fréquemment multiples.

\section{Les soins familiaux}

L'espace familial est le principal espace thérapeutique où la plupart des enfants cherchent et trouvent des soins et du soulagement à leurs maux de ventre. L'espace familial se limite surtout aux personnes habitant dans une même maison, là où la mère joue toujours le rôle principal. Parfois, elle est secondée par les autres membres de la fratrie et par la famille élargie. L'univers domestique est le lieu de départ des itinéraires thérapeutiques de ces familles. C'est l'espace où s'élabore le plan de la thérapie, à savoir, les consensus et les décisions sur ce qu'il faut faire et où se rendre (Saillant, 1999).

Par ailleurs, une sorte d'itinéraire thérapeutique est suivi au sein même de ces familles. D'abord, quand les enfants ressentent des douleurs au ventre, ils pensent que ces maux passeront d'eux-mêmes, au bout de quelque temps, sans qu'il y ait à faire ou à dire. En général, les enfants cessent l'activité du moment comme jouer. Ensuite, ils boivent de l'eau ou vont à la toilette. Au tout début, ils ne disent à personne qu'ils ont des maux de ventre et espèrent que ces douleurs passeront rapidement, sans qu'ils aient recours à de l'aide ou sans entreprendre une action quelconque :

«Je n'ai rien fait, juste j'étais assis à regarder la télé, et ça a passé.» (Louis, douze ans) 
Toutefois, dans la mesure où les maux de ventre persistent, les enfants entreprennent d'autres actions pour les soulager. Ces dernières sont principalement entreprises au sein de l'espace familial et, selon le point de vue des mères et des enfants, elles obéissent à une certaine chronologie selon leur efficacité à soulager le mal. D'abord, les enfants vont à la toilette et ils essaient de déféquer; ils peuvent aussi aller se coucher ou essayer de se réchauffer. D'autres enfants confient toujours à leur mère qu'ils ont des maux de ventre et se rapprochent d'elle.

En apprenant que leurs enfants ont des maux de ventre, les mères mettent en marche quelques actions visant à les soulager, comme leur préparer un thé, leur donner un médicament, faire des prières, leur faire des massages sur le ventre ou leur donner quelque aliment. Dans le même sens que les conclusions avancées par Desclaux (1996, p. 255) par rapport au rôle des mères dans les modèles explicatifs des maladies infantiles, nous avons trouvé que ce sont elles qui, en décryptant les signes et en ordonnant les symptômes, choisissent une attitude, un recours, et appliquent une thérapeutique. En s'occupant ainsi du malade et en prenant la décision du type d'aide auquel recourir, ces mères pourraient être considérées comme faisant partie du therapy management group.

"Du point de vue de la plupart des mères et des enfants, le fait de se rapprocher d'eux et de leur donner de l'affection contribue au soulagement de leurs maux de ventre..."
Du point de vue de la plupart des mères et des enfants, le fait de se rapprocher d'eux et de leur donner de l'affection contribue au soulagement de leurs maux de ventre :

"J'appelle ça une sorte de rapprochement. Le plus souvent, quand elles ne se sentent pas bien, elles viennent auprès de moi et elles me disent: «Maman, je ne me sens pas bien», et je dis : "Qu'est-ce que ma fille a?» J'essaie de donner quelque chose pour soulager la douleur... On est bien obligé de faire quelque chose. Parfois, on s'approche plus d'eux pour leur donner de l'affection, et pour trouver des moyens thérapeutiques pour soulager la douleur." (mère de la famille Roney) 
"Dans mes coutumes à moi... qu'on connaît de nos parents, de nos grands-parents. Quand il y a quelqu'un qui a mal au ventre, on lui donne du thé... la feuille d'orange, on fait bouillir et on donne aux enfants... La feuille de cachima, c'est pour quand vous avez mal au ventre... donc, on en fait. " (mère de la famille Cadet). »
Le réchauffement corporel représente une autre ressource habituellement envisagée et utilisée par ces mères. Il y a différentes actions qui peuvent conduire à réchauffer le ventre et le corps de ces enfants. Ainsi, quelques-uns parlent de l'acte de se coucher, de se rapprocher physiquement de leur mère, de se faire faire des massages sur leur ventre ou encore de boire du thé chaud ou de manger de la soupe.

Le thé est aussi utilisé par la plupart des familles pour ses propriétés médicinales. Toutes les mères, comme la majorité des enfants, pensent qu'en effet le thé les aide à soulager le mal de ventre et elles l'utilisent dès l'apparition des premiers symptômes. Le thé est désigné sous son nom générique de «thé "; cependant, on peut distinguer le thé à base de feuilles d'orange et de cachima, le thé au citron, au miel et au sel, le thé avec des feuilles amères ou à base de camomille et le thé avec du sel. En général, toutes ces mères préparent ces thés et autres remèdes selon les méthodes apprises durant leur jeunesse en Haïti ou encore grâce à des contacts qu'elles gardent toujours avec des proches vivant là-bas ou ailleurs, et aussi en parlant avec quelques migrants haïtiens plus âgés de leur voisinage :

«Dans mes coutumes à moi... qu'on connaît de nos parents, de nos grands-parents. Quand il y a quelqu'un qui a mal au ventre, on lui donne du thé... la feuille d'orange, on fait bouillir et on donne aux enfants.... La feuille de cachima, c'est pour quand vous avez mal au ventre.... donc, on en fait. " (mère de la famille Cadet).

Quelques familles ont aussi parlé de prendre des mesures préventives ou correctives telles la suppression de certains aliments ou la consommation d'autres de nature opposée.

La plupart des membres des familles considèrent que le fait de manger des aliments sucrés peut causer des maux de ventre. Par conséquent, ces mères essaient de faire en sorte que leurs enfants n'en mangent pas trop ou n'en mangent pas du tout quand ils éprouvent de telles douleurs. En revanche, elles leur donnent à manger des aliments salés ou à boire de l'eau avec 
"...le recours aux médicaments en vente libre est une ressource complémentaire aux autres moyens utilisés. » du sel. Également, elles essaient de prévenir ces maux de ventre en donnant aux enfants un aliment consistant et salé, surtout le matin, avant qu'ils quittent pour l'école.

Pour les enfants, dans la majorité des cas, les maux de ventre passent après s'être couchés, s'être réchauffés ou après avoir bu une boisson chaude. Néanmoins, il y a des situations où les douleurs persistent. Les mères envisagent alors d'autres sources de soulagement, comme donner un médicament à leurs enfants. Ainsi, le recours aux médicaments en vente libre est une ressource complémentaire aux autres moyens utilisés. Parmi les médicaments nommés par ces familles, on trouve le Tylenol, le Pepto Bismol et l'Advil. En général, les enfants ont commencé à prendre ces médicaments dès que les médecins consultés les leur ont prescrits. Par la suite, leur mère a continué à les utiliser comme une sorte d'automédication, sans avoir besoin de se rendre encore une fois chez le médecin. En outre, les infirmières de l'école de quelques enfants leur ont conseillé de prendre ces médicaments.

\section{Une dynamique de soins qui s'installe dans l'espace familial}

Mis à part les soins prodigués et les remèdes préparés à la maison, par les mères surtout, une certaine dynamique sociale s'installe au sein des familles nucléaires quand les enfants ont mal au ventre. Cette dynamique se présente d'une manière particulière dans chaque famille, en fonction de sa composition, du rôle de chacun de ses membres, de l'intensité des maux de ventre, des autres symptômes ressentis par les enfants et des ressources sur lesquelles les mères peuvent compter. Cependant, on peut dire que les relations entre les membres de la famille nucléaire et les rôles qui leur sont assignés changent durant ces épisodes de maux de ventre, notamment quand ils deviennent plus forts. À ce sujet, on a déjà mentionné que le principal changement sur le plan des relations entre les membres de la famille nucléaire tient d'une proximité physique et affective qui s'installe entre les mères et leurs enfants. D'autres changements au sein des familles sont liés au fait que les mères permettent aux enfants malades de se reposer 
"Quand les enfants ont des maux de ventre à l'école, et surtout quand ceuxci ne passent pas rapidement ou qu'ils sont intenses, ils en parlent aux professeurs ou à l'infirmière. Pas tant pour obtenir de l'aide que la permission de s'en aller chez eux..." et d'accomplir moins de tâches que d'habitude, voire, d'en être totalement dispensés.

Quand les enfants ont des maux de ventre à l'école, et surtout quand ceux-ci ne passent pas rapidement ou qu'ils sont intenses, ils en parlent aux professeurs ou à l'infirmière. Pas tant pour obtenir de l'aide que la permission de s'en aller chez eux :

«On le dit juste au prof pour nous faire... une réparation... pour partir à la maison. On va voir une secrétaire pour dire qu'on veut rentrer à la maison, et pour qu'on appelle les parents... Les professeurs, c'est juste quand ils ne veulent pas me laisser sortir de la classe, à part ça, il n’y pas de besoin.» (Augustin, douze ans)

Quelques enfants ont tendance à se replier sur eux-mêmes et sur leur famille nucléaire, en faisant de leurs problèmes de ventre une partie de leur vie privée. Par exemple Augustin qui déclare :

«On le garde ça secret... des fois on ne dit pas même au professeur..."

Et ses deux sœurs qui préferent aussi ne pas en parler, craignant d'être signalées comme porteuses d'une maladie contagieuse :

«Parce qu'il y a des gens qui le prennent mal, il y a des gens qui disent: "Ah! La personne a des microbes...» Des fois je ne dis pas... Je peux avoir mal au bras, mais je ne vais pas le dire, parce que... je n'aime pas ça.»

Quand un enfant malade rentre chez lui, la maison devient une sorte d'hôpital où chaque membre de la famille s'implique pour le soigner :

«Des fois, si c'est elle [Caméla] qui est malade, c'est elle [Christine] qui est l'infirmière... Si c'est le gars, les deux filles sont les infirmières. Ils ont beaucoup d'approches... Moi, je suis le boss. Moi, quand ils sont... je peux dire que la 
journée, je dois rester auprès des enfants pour donner un peu de soins comme maman.» (mère de la famille Pétrus)

Cette famille a tendance à se replier sur elle-même au moment de communiquer et de chercher un soulagement aux problèmes de ventre de ses enfants. Présente surtout chez les jeunes, cette tendance est toutefois basculée et nuancée par leur mère, dans la mesure où elle établit une communication avec quelques personnes de l'extérieur, comme les infirmières de l'école, les conseillers d'Info-santé ou les médecins des services d'urgence.

De même façon, les autres familles essaient presque toujours de gérer et de soulager elles-mêmes les maux de ventre et les autres problèmes qui y sont liés, en faisant de ces problèmes une partie de leur vie privée. Néanmoins, ces maux ne sont pas strictement circonscrits à l'espace familial, ils sont aussi canalisés à travers quelques réseaux sociaux qui jouent entre leur vie privée et leur vie publique.

«Parmi les principales ressources utilisées, nous trouvons quelques pratiques religieuses, le soutien et la socialisation avec des personnes rencontrées dans les églises fréquentées par ces familles. Les plus vieux immigrants haitiens habitant à Montréal sont également des ressources utilisées par certaines des familles que nous avons rencontrées. "

\section{Les ressources religieuses et communautaires}

Dans leur quête de soulagement des maux de ventre dont souffrent leurs enfants, les familles utilisent différentes ressources de façon complémentaire. Il y a une tendance à chercher de l'aide au sein des espaces thérapeutiques alternatifs plus fréquemment qu'au sein de la médecine officielle. Parmi les principales ressources utilisées, nous trouvons quelques pratiques religieuses, le soutien et la socialisation avec des personnes rencontrées dans les églises fréquentées par ces familles. Les plus vieux immigrants haïtiens habitant à Montréal sont également des ressources utilisées par certaines des familles que nous avons rencontrées.

Les pratiques religieuses consistent principalement à faire des prières, individuelles ou collectives, et à participer à des conférences sur la santé en général. En effet, d'après ces familles, les maux de ventre dont souffrent leurs enfants ne constituent pas nécessairement un problème à traiter d'une façon isolée des autres problèmes de santé et des besoins familiaux. Questionnées sur le rôle que joue la religion pour soulager ces maux de ventre, 
"...on peut envisager

les savoirs médicaux de ces familles immigrantes comme des stratégies sociales et individuelles qu'elles développent. De telles stratégies portent sur les soins et la promotion de la santé, la compréhension des maladies et des malaises, leur traitement, la prévention et la guérison; de même, elles sont parfois orientées vers la création et la reproduction de liens de socialisation et de solidarité. » les mères pensent que les prières et la foi en Dieu aident à guérir autant ces maux que tout autre problème de santé :

"On prie, et Dieu fait quelque chose. Parce que j'ai confiance en lui. Je ne fais pas de confiance en personne, c'est lui qui est capable de faire tout. " (mère de la famille Chauvet)

"Quand nous sommes malades, avant d'aller voir le médecin, nous prions au Bon Dieu. Je crois que Dieu seul peut me guérir, et ensuite le médecin avec son médicament peut m'aider.» (mère de la famille Roney)

Également, toutes ces familles disent réciter des prières collectivement dans le cadre de célébrations à l'église. En général, de telles prières ont pour but de demander la guérison des problèmes de ventre chez les enfants et d'autres problèmes de santé de la famille.

En sus des pratiques religieuses, les mères demandent aussi l'aide de personnes qui travaillent dans des organismes communautaires qu'elles fréquentent, d'amis qui fréquentent l'église ou d'autres immigrants haïtiens, de Montréal ou d'ailleurs. Ceux-ci conseillent de baisser la température corporelle et aident même à baigner les enfants :

«Les Haïtiens qui sont plus âgés que nous... pour nous expliquer... Des fois, ils nous mettent dans le bassin... On fait savonner, pour rafraîchir.» (mère de la famille Pétrus)

Ainsi, on peut envisager les savoirs médicaux de ces familles immigrantes comme des stratégies sociales et individuelles qu'elles développent. De telles stratégies portent sur les soins et la promotion de la santé, la compréhension des maladies et des malaises, leur traitement, la prévention et la guérison; de même, elles sont parfois orientées vers la création et la reproduction de liens de socialisation et de solidarité. D'après Taboada-Leonetti (1994), un des niveaux d'expression des stratégies des immigrants, et qui comptent parmi les plus accessibles, se trouve dans le domaine culturel; il s'exerce 
"Les familles qui ont consulté les services médicaux sont généralement insatisfaites des réponses données par les médecins en matière d'explication et de guérison des maux. Une telle insatisfaction a renforcé la tendance qu'ont les familles à ne pas consulter fréquemment les services formels de médecine." dans la sphère du privé, au sein de la famille ou de la communauté. Ainsi, la communauté minoritaire tentera d'affirmer son existence à travers des "stratégies de contournement ": de la religion ou de pratiques culturelles quotidiennes comme les soins de santé et les rituels de guérison.

\section{Le recours à la médecine officielle}

La médecine officielle constitue également une des ressources utilisées par ces familles dans la recherche du soulagement des maux de ventre des enfants. La plupart du temps, ces familles se sont dirigées vers les urgences des hôpitaux. À part ces consultations, elles n'ont pas suivi de traitements médicaux particuliers traitant des problèmes de ventre des enfants. Les familles qui ont consulté les services médicaux sont généralement insatisfaites des réponses données par les médecins en matière d'explication et de guérison des maux. Une telle insatisfaction a renforcé la tendance qu'ont les familles à ne pas consulter fréquemment les services formels de médecine. En même temps, ces familles ne perçoivent pas les maux de ventre de leurs enfants comme une maladie. Cependant, cette dynamique est complexe et paradoxale dans certains cas. Les familles oscillent toujours entre le désespoir et la confiance aux médecins, ceux-ci n'ayant pas confirmé l'existence d'une maladie chez les enfants. Elles ont tendance à rechercher des explications et des solutions dans d'autres espaces thérapeutiques, considérant souvent la médecine officielle comme leur dernier recours quand les maux de ventre dépassent la capacité de tolérance et de gérance des enfants et de leurs mères. Mais en même temps, ces familles perçoivent les médecins comme des personnes qui détiennent le savoir et le pouvoir nécessaires pour faire face à ces douleurs. Ainsi, elles ne nient pas la capacité des cliniciens de comprendre et de résoudre efficacement ces douleurs à d'autres moments données.

Les enfants rencontrés n'ont pas de maux de ventre qu'ils ne peuvent supporter ou qui débordent la capacité de gestion et de contrôle de leur mère. Par conséquent, ces familles ont tendance à considérer que ces douleurs ne constituent pas un problème de santé justifiant un recours médical. Parfois, les 
«Certaines

expériences passées teintent également le rapport des mères avec les services médicaux. Elles disent avoir été victimes de services médicaux négligents et de traitements presque inhumains." enfants éprouvent d'autres douleurs et d'autres malaises qui sont plus intenses et dérangeants que les maux de ventre. Il en est de même pour leurs familles, qui perçoivent et interprètent ces autres symptômes comme plus inquiétants et prioritaires. Ainsi, les maux de ventre sont placés au second plan et ils sont traités dans l'espace thérapeutique familial ou avec des ressources autres que celles de la médecine officielle.

À défaut d'un diagnostic clair et de la guérison des maux de ventre de ces enfants, les familles ont d'autres plaintes par rapport aux services médicaux. Ces plaintes portent, d'un côté, sur les longues heures d'attentes qu'elles doivent passer dans les services d'urgence pour obtenir un rendez-vous et, d'un autre côté, sur la mauvaise qualité des soins reçus. Quoique ces plaintes ne se trouvent pas parmi les motifs qui ont empêché ces familles de se rendre chez le médecin, et qu'elles n'ont pas un rapport direct avec les consultations pour les maux de ventre, ces attentes sont rapportées comme très démotivantes et décourageantes.

Certaines expériences passées teintent également le rapport des mères avec les services médicaux. Elles disent avoir été victimes de services médicaux négligents et de traitements presque inhumains. La mère de la famille Roney, par exemple, déclare avoir beaucoup souffert à cause de la négligence des médecins et du personnel médical à Port-au-Prince. De plus, de telles expériences se trouvent parmi les principaux motifs qui l'ont amenée à prendre la décision de quitter Haïti pour le Canada. Cette mère n'a pas consulté les médecins de Montréal parce qu'elle s'aperçoit que les maux de ventre de ses filles ne sont pas vraiment aigus. Catherine etVictoire, de leur côté, pensent que les médecins pourraient les aider à soulager leurs douleurs, et par conséquent, elles aimeraient les consulter, surtout Catherine, l'aînée.

\section{Conclusion}

Les multiples explications et significations que les familles attribuent aux maux de ventre de leurs enfants démontrent que cette interprétation se trouve dans un processus de 
"Certains immigrants reconstruisent des univers de sens en s'éloignant des paramètres fournis par leur société d'origine, d'autres adoptent les manières prévalant dans la société d'accueil, la majorité des familles fabriquent des systèmes de sens plus ou moins créolisés, en combinant des éléments issus de différentes matrices culturelles...» reconfiguration constante, où plusieurs significations se partagent la scène. Connaitre ces dynamiques est important afin de ne pas surculturaliser les interprétations des patients et des familles immigrantes et surinvestir la culture dans une situation clinique. Dans ce contexte, l'anthropologie nous enseigne que l'universalité, malgré toutes les apparences, ne peut être postulée d'emblée à partir d'une position a priori. Ainsi, on ne peut présupposer ni le lieu où se tient la famille, ni la configuration de leur système de sens par rapport à la maladie. Certains immigrants reconstruisent des univers de sens en s'éloignant des paramètres fournis par leur société d'origine, d'autres adoptent les manières prévalant dans la société d'accueil, la majorité des familles fabriquent des systèmes de sens plus ou moins créolisés, en combinant des éléments issus de différentes matrices culturelles - par exemple, la culture d'origine et la culture locale. Les professionnels doivent ainsi dépasser la folklorisation et l'étiquetage ethnique qui réduisent souvent les familles d'autres origines ethniques à quelques croyances ou comportements stéréotypés. Il s'agit donc d'un apprentissage visant à reconfigurer la carte mentale pour une meilleure adéquation des services de santé à la diversité culturelle.

Nous avons vu que les douleurs de ventre chez ces enfants sont canalisées à travers l'espace familial et les réseaux sociaux, représentés par des organismes communautaires et des églises. Tenir compte des ressources sociales et symboliques des personnes, ainsi que de leurs dimensions sociales et culturelles, aide à avoir une meilleure compréhension du contexte dans lequel ces problèmes de santé se produisent. Nous pensons qu'il est important de mettre en place un cadre complexe inspiré d'une lecture sociale et culturelle des situations cliniques et de situer la maladie dans un contexte relationnel. En ce qui concerne les familles immigrantes, comme celles d'Haïti, les parents et leurs enfants vivent souvent au carrefour de plusieurs univers culturels.

En outre, les explications et ressources sur lesquelles comptent ces familles pour faire face aux maux de ventre de leurs enfants sont multiples. Elles témoignent également du rôle actif que ces familles et ces mères jouent sur la santé des enfants. Tenir compte de ces dynamiques familiales constitue un outil important qui 
"Il est important

d'avoir une

connaissance

approfondie de la qualité des relations

médicales et des

transactions entre les

cliniciens, les enfants

malades et leurs

familles, ainsi que

l'adoption des modèles d'ajustement culturel

de la prise en charge et des soins qui soient significatifs dans le monde des familles. " permet aux professionnels de la santé de mieux agir sur le plan de la prévention et de la promotion de la santé des enfants. De plus, connaitre les différentes explications qui selon les familles sont à la base des maux de ventre - par exemple, le type d'aliment consommé et la température - permet de définir plus clairement un plan de promotion et de prévention de la santé qui tient compte des facteurs de vulnérabilité et de protection. De même, prendre connaissance des ressources sociales et matérielles sur lesquelles s'appuient ces familles favorise la mise en place de stratégies complémentaires pour traiter ces douleurs et d'autres problèmes de santé chez ces enfants.

Enfin, ces familles ont des opinions ambivalentes par rapport aux services médicaux, dans la mesure où les diagnostics et les remèdes fournis par les médecins ne sont pas satisfaisants la plupart du temps. Notre travail anthropologique a permis de documenter ce que les parents et leurs enfants retiennent de la rencontre clinique, des conseils suggérés et leur impression d'être entendus à divers degrés. Il est important d'avoir une connaissance approfondie de la qualité des relations médicales et des transactions entre les cliniciens, les enfants malades et leurs familles, ainsi que l'adoption des modèles d'ajustement culturel de la prise en charge et des soins qui soient significatifs dans le monde des familles. Il est essentiel que les professionnels disposent de meilleurs instruments pour accéder aux systèmes de sens, dans les situations et conditions vécues par les personnes et les familles immigrantes. Notre recherche a apporté quelques éléments dans ce sens-là, éléments que nous espérons continuer à développer.

\section{Note}

Afin de protéger l'identité des répondantes et répondants, les noms et prénoms servant à les identifier dans cet article sont fictifs.

\section{Bibliographie}

AR GANT-LE CLAIR, Marie-Claude (1991). Primiparentalité et rites de passage chez des couples québécois et haïtiens, Montréal, Université de Montréal, 996 p. 
BIBEAU, Gilles, et collab. (1992). La Santé mentale et ses visages : un Québec pluriethnique au quotidien, Montréal, Gaëtan Morin éd. et Comité de la santé mentale du Québec, 289 p.

BIBEAU, Gilles (1987). À la fois d'ici et d'ailleurs : les communautés culturelles du Québec dans leurs rapports aux services sociaux et aux services de santé, Québec, Publications du Québec, 213 p.

BIJOUX, Legrand (1990). Coup d'oeil sur la famille haïtienne, Port-au-Prince, Éditions des Antilles, $135 \mathrm{p}$.

BRODWIN, Paul (1996). Medicine and morality in Haït : the contest for healing power, New York, Cambridge University Press, 260 p.

CATHÉBRAS, Pascal, et collab. (2006). Facteurs étiologiques de la somatisation et des symptômes fonctionnels, Paris, Masson, $240 \mathrm{p}$.

COMPÈRE, Lejacques, et George-Marie CRAAN (1995). « De la fragilisation à la marginalisation. Un regard critique sur la communauté haïtienne de Montréal », PRISME, vol.5, n 1, p. 6877.

CORIN, Ellen, et Gilles BIBEAU (1995). «Culturaliser l'épidémiologie psychiatrique. Les systèmes des signes, de sens et d'actions en santé mentale ", dans François Trudel (dir.), La construction de l'anthropologie québécoise, Québec, Les Presses de l'Université Laval, p 105-148.

DENZIN, Norman K., et Yvonna S. LINCOLN (dirs.) (2000). Handbook of qualitative research, Thousand Oaks, Sage Publications, 1065 p.

DESCLAUX, Alice (1996). "De la mère responsable et coupable de la maladie de son enfant », dans Jean Benoist, Soigner au pluriel, Paris, Éditions Karthala, p. 251 -280.

INSTITUT DE LA STATISTIQUE QUÉBEC (ISQ) (2009). http://www.stat.gouv.qc.ca/publications/referenc/quebec_stat/con_rev/con_rev_7.htm,

JANZEN, John M. (1987). «Therapy Management : concept, reality, process », Medical anthropology quarterly, New Series, vol.1, no 1, p. 68-84.

KANASAWA, Motoyori, et collab. (2004). «Patients and nonconsulters with irritable bowel syndrome reporting a parental history of bowel problems have more impaired psychological distress ", Digestive Diseases and Sciences, Vol. 49, No 6 p. 1046-1053.

KLEINMAN, Arthur (1988). The illness narratives : suffering, healing, and the human condition, New York, Basic Books, 304 p.

LAGUERRE, Michel (1987). Afro-Caribbean folk medicine, Massachusetts, Bergin \& Garvey, 256 p.

LEVY, Rona L., et collab. (2006). "Psychosocial aspects of the functional gastrointestinal disorders ", Gastroenterology, Vol. 130, № 5, p 1447-1458.

LINDLEY, Keith J., et collab. (2005). " Consumerism in healthcare can be detrimental to child health : lessons from children with fonctionnal abdominal pain ", Archives of Disease in Childhood, Vol. 90, p. 335-337.

MASUD, Mohammed A., et collab. (2001). "Irritable bowel syndrome in a rural community in Bangladesh : prevalence, symptoms pattern and health care seeking behaviour ", American Journal of Gastroenterology, Vol. 96, № 5, p. 1547-1552.

MASSÉ, Raymond (1995). Culture et santé publique : les contributions de l'anthropologie à la prévention et à la promotion de la santé, Montréal, Gaëtan Morin, $499 \mathrm{p}$.

MAYER Robert, et collab. (2000). Méthodes de recherche en intervention sociale, Montréal, Gaëtan Morin, $410 \mathrm{p}$. 
OKEKE ,Edith N., et collab. (2005). «Prevalence of irritable bowel syndrome in a Nigerian student population ", African Journal of Medicine and Medical Sciences, Vol. 34, № 1, p. 33-36.

OLUBUYIDE, IO, et collab. (1995). "A study of irritable bowel syndrome diagnosed by Manning criteria in an African population ", Digestive Diseases and Sciences, Vol. 40, No. 5, p. 983-985.

PIERRE-JACQUES, Charles, et collab. (dirs.) (1982). Actes du Colloque sur l'enfant haïtien en Amérique $d u$ Nord : santé, scolarité, adaptation sociale, Montréal, Centre de recherches caraibes, Université de Montréal, 132 p.

PIERRE-JACQUES, Charles (1980). Le jeune haïtien et l'école québécoise, Montréal, Centre de recherches caraibes, Université de Montréal, $93 \mathrm{p}$.

POUPART, Jean, et collab. (1997). La recherche qualitative : enjeux épistémologiques et méthodologiques, Montréal, Gaëtan Morin, 405 p.

SAILLANT, Francine (1999). "Femmes, soins domestiques et espace thérapeutique ", Anthropologie et Sociétés, Vol. 23, n², p. 15-40.

STATISTIQUES CANADA (2009). " Recensement de 2006 ", réf. du 28 décembre 2009, http://www12.statcan.ca/censusrecensement/

TABOADA-LEONETTI, Isabelle (1994). "Stratégies identitaires et minorités dans les sociétés pluriethniques ", Revue internationale d'action communautaire, Vol. 21, № 61, p. 95-108.

TALLEY Nicholas J., et Robin SPILLER (2002). «Irritable bowel syndrome : a little understood organic bowel disease? ", Lancet, Vol. 360, No 9332, p. 555-564. 